\title{
Bariatric Patient Experience with Virtual Care During the COVID-19 Pandemic
}

\author{
Krista Hardy ${ }^{1}$. Anastasia Anistratov ${ }^{1} \cdot$ Wenjing He ${ }^{1} \cdot$ Felicia Daeninck ${ }^{1} \cdot$ Jeffrey Gu² $\cdot$ Ashley Vergis in $^{1,3}$
}

Received: 23 July 2021 / Revised: 5 November 2021 / Accepted: 23 November 2021 / Published online: 4 January 2022

(c) The Author(s), under exclusive licence to Springer Science+Business Media, LLC, part of Springer Nature 2021

The ongoing COVID-19 pandemic imposed substantial disruption to the provision of routine outpatient care [1]. Declaration by the World Health Organization (WHO) prompted health care professionals to restrict in-person interactions to urgent and essential services [2]. Hospitals canceled scheduled elective surgeries to ensure enough hospital resources were available to accommodate COVID-19 patients [3]. These restrictions catalyzed the implementation of telehealth services by physicians and allied health services to limit the risk of disease transmission while aiming to provide patients with continuous accessible care [3].

The Center for Metabolic and Bariatric Surgery (CMBS) is the sole bariatric surgery program located in Manitoba, Canada. At the outset of the pandemic, the CMBS postponed elective bariatric procedures and shifted all routine postoperative follow-ups from in-person to virtual telephone appointments in compliance with government and public health official orders. At this time, all postoperative followup appointments continue to be conducted by telephone unless there is a specific indication for in-person clinical assessment (i.e. drain removal, significant symptoms arising due to surgery). Typically, the CMBS program requires patients to attend pre and postoperative assessments along with a behavior change program, providing patients support, education and resources to promote healthy lifestyle choices. Patients attend follow-up appointments postoperatively for

Authors Krista Hardy and Anastasia Anistratov have contributed equally to this work.

\section{Ashley Vergis}

umserg99@gmail.com

1 Department of Surgery, University of Manitoba, Winnipeg, Manitoba, Canada

2 Community Health and Epidemiology, Department of General Surgery, University of Saskatchewan, Saskatoon, SK, Canada

3 St. Boniface General Hospital, Z3039-409 Tache Avenue, Winnipeg, MB R2H 2A6, Canada up to 5 years where surgeons assess weight loss and comorbidity resolution and continue to provide resources and support to patients to ensure proper weight loss is maintained. Therefore, postoperative appointments are essential to the weight loss, weight loss maintenance and long-term selfmanagement of obesity [4].

Due to its rapid implementation, we lack insight into the effects of virtual care on the postoperative bariatric population. Therefore, we developed a patient-reported experience measure (PREM)-based questionnaire to explore patient experiences and satisfaction with postoperative telephone follow-up compared to previous in-person appointments with a bariatric surgeon. Questions addressing overall satisfaction, access to technology, call quality, future follow-up preferences, time and cost constraints were explored using a 5-point Likert Scale. Additional questions addressing particular likes/dislikes of virtual follow-up, approximate travel and waiting times, and costs associated with in-person and virtual follow-up were explored through multiple select questions.

One hundred thirty-eight patients who were ( $>18$ years) enrolled in the CMBS program; who underwent gastric bypass or sleeve gastrectomy at the CMBS; and had received both in-person and virtual postoperative follow-up appointments with a bariatric surgeon agreed to participate in the survey. Of the 138 surveys distributed, 101 respondents completed the online questionnaire (73.2\% response rate).

We found that majority of patients $(81.7 \%)$ were satisfied with their virtual follow-up, mainly due to the alleviation of both time and cost constraints/concerns associated with attending their virtual care appointment (Table 1). Most (96.9\%) virtual follow-up appointments kept estimated cost expenditures such as parking, gas and wages lost at $\$ 0-10$ compared to half $(51.0 \%)$ of in-person appointments (Table 2). Waiting times were significantly less for virtual appointments. Most (59.8\%) patients waited only 0-5 min before their virtual follow-up began, while $44.4 \%$ waited 15-30 min before starting their in-person appointment 
Table 1 Cost constraints and patient satisfaction associated with virtual follow-up

\begin{tabular}{llllll}
\hline & Strongly agree & Agree & $\begin{array}{l}\text { Neither agree or } \\
\text { disagree }\end{array}$ & Disagree & $\begin{array}{l}\text { Strongly } \\
\text { disagree }\end{array}$ \\
\hline $\begin{array}{l}\text { Less cost constraints } \\
\text { with virtual follow- }\end{array}$ & 50.5 & 29.9 & 15.5 & 3.10 & 1.00 \\
$\quad \begin{array}{l}\text { up: (\%) } \\
\begin{array}{l}\text { Satisfied with virtual } \\
\text { follow-up: (\%) }\end{array}\end{array}$ & 37.8 & 43.9 & 15.3 & 3.00 & 0 \\
\hline
\end{tabular}

Table 2 Time/cost expenditures and future follow-up preferences associated with in-person and virtual appointments

\begin{tabular}{llc}
\hline & Virtual & In-person \\
\hline Waiting time: $(\%)$ & & \\
0-5 min & 59.8 & 5.10 \\
5-15 min & 17.5 & 40.4 \\
15-30 min & 8.20 & 44.4 \\
30-45 min & 2.10 & 9.10 \\
$>$ 45 min & 12.4 & 1.0 \\
Time spent during appointment: (\%) & & \\
0-15 min & 90.7 & 75.7 \\
$>15$ min & 9.30 & 24.3 \\
Travel time: $(\%)$ & & \\
<30 min & 94.8 & 39.4 \\
$>$ 30 min & 5.20 & 60.0 \\
Cost expenditure: $(\%)$ & & \\
\$0-10 & 96.9 & 51.0 \\
\$10-30 & 1.10 & 24.6 \\
\$30-75 & 1.00 & 12.2 \\
\$75-200 & 1.00 & 6.10 \\
$>$ \$200 & 0 & 6.10 \\
Future follow-up preference: $(\%)$ & 76.0 & 24.0 \\
\hline
\end{tabular}

(Table 2). Additionally, $60.6 \%$ of in-person follow-ups spent over $30 \mathrm{~min}$ traveling to their appointment while $84.5 \%$ of virtual appointments were associated with no travel time at all. A review by Nguyen et al. on patient satisfaction with telemedicine paralleled our findings, stating patient satisfaction was primarily influenced by time and cost-effectiveness of synchronous visits offered by various health services [5]. Further analysis of patient preferences revealed that $76.0 \%$ of patients preferred virtual care as an option for future follow-up appointments $(52.0 \%$ telephone; $21.3 \%$ video (i.e. Microsoft Teams, Zoom); $2.7 \%$ telehealth (utilizing a teleconference system). This might shift as the COVID-19 pandemic ends, though it is reasonable to expect virtual care will be one of the long-standing changes in health care delivery.

Additionally, majority of the non-urban population preferred ongoing virtual care and were satisfied with virtual appointments overall $(64.7 \%)$. The responses align with the knowledge that telehealth services have made health care more accessible and convenient to patients living in remote/rural communities by alleviating the cost and time constraints of travel [6-8]. Despite majority of patients being satisfied with their virtual experiences and $49.5 \%$ indicating no major concerns associated with their virtual care experience (such as absence of/difficulty using technology, potential lack of privacy or insufficient assessment), we would like to mention that over a third of respondents (35.1\%) stated they disliked not being able to see the surgeon in-person.

While advanced patient age, lower level of education and limited computer literacy have been reported as potential barriers to adopting telemedicine [9], at the time of data collection, a secure video platform such as Microsoft Teams was not available to surgeons at the CMBS to conduct follow-up appointments. Therefore, the survey solely addressed virtual telephone experiences, eliminating technological barriers associated with the use of video platforms. Additionally, majority of our survey respondents $(66.3 \%)$ were between 40 and 60 years of age at the time of surgery and $86.7 \%$ of all respondents were female, characteristic of a general bariatric population (Table 3). Given the narrow age range of our patient population (29-63 years) in addition to bariatric surgery rarely being performed on patients of advanced age ( $>65$ years), age is unlikely to be a major barrier in the access and use of virtual care in this population.

We would also like to note that $8.5 \%$ respondents selfidentified as Indigenous (First Nation, Métis, Inuit). Of the urban Indigenous study population, preference was shown for ongoing in-person care. Face-to-face health care interactions are valued by Indigenous peoples, and culturally sensitive and trauma-informed care can enhance health outcomes and experiences in this patient population [10]. Further exploration is warranted to understand the utility and acceptability of telehealth in Indigenous day-to-day life given the health inequities arising from the effects of colonization, including disparities in the social and Indigenous determinants of health, cultural oppression, and all forms of racism and intergenerational trauma that contribute to the health care experiences of Indigenous peoples $[10,11]$.

Recently, Microsoft Access has been approved for synchronous visits in the CMBS program, and is 
Table 3 Patient demographics

\begin{tabular}{|c|c|}
\hline Demographics (\%) & Frequency \\
\hline \multicolumn{2}{|l|}{ Sex: $n(\%)$} \\
\hline Female & 86.7 \\
\hline Male & 13.3 \\
\hline \multicolumn{2}{|l|}{ Age group: (\%) } \\
\hline $20-29$ & 2.10 \\
\hline $30-39$ & 22.1 \\
\hline $40-49$ & 35.8 \\
\hline $50-59$ & 30.5 \\
\hline $60-69$ & 9.50 \\
\hline \multicolumn{2}{|l|}{ Ethnicity: (\%) } \\
\hline Caucasian & 84.2 \\
\hline First Nation & 1.10 \\
\hline Metis & 7.40 \\
\hline Asian & 2.10 \\
\hline Hispanic & 2.10 \\
\hline Do not know & 1.00 \\
\hline Other & 2.10 \\
\hline \multicolumn{2}{|l|}{ Education: (\%) } \\
\hline Less than high school & 1.00 \\
\hline Completed high school & 17.9 \\
\hline Some technical or community college & 16.8 \\
\hline Completed technical or community college & 24.2 \\
\hline Some university & 7.40 \\
\hline Bachelor's degree & 26.3 \\
\hline Master's degree & 3.20 \\
\hline Professional degree or doctorate & 3.20 \\
\hline \multicolumn{2}{|l|}{ Residence: (\%) } \\
\hline Urban & 81.7 \\
\hline Rural & 18.3 \\
\hline \multicolumn{2}{|l|}{ Type of surgery: (\%) } \\
\hline $\mathrm{RYGB}^{\mathrm{a}}$ & 90.5 \\
\hline $\mathrm{LSG}^{\mathrm{b}}$ & 6.30 \\
\hline Unknown & 3.20 \\
\hline
\end{tabular}

${ }^{\mathrm{a}} R Y G B$ Roux-en-Y gastric bypass, ${ }^{\mathrm{b}} L S G$ laparoscopic sleeve gastrectomy

anticipated to be implemented as an option for routine postoperative care. Going forward, we would like to investigate patient satisfaction with the option of selecting telephone versus video format. We plan to assess the outcome of offering a patient-selected option for followup, recognizing that some patients may have constraints associated with video appointments (i.e. internet access, lack of software familiarity). This will help address the question of patient preferences and benefits surrounding in-person versus virtual care in a post-pandemic setting. The efficacy of virtual patient care in terms of missed patient concerns and/or medical complications should be considered. Provider experience including time savings, user fatigue and perceptions of adequacy of virtual assessments and surgeon satisfaction with telephone versus video appointments should also be evaluated.

Despite the rapid transition from in-person to virtual care, majority of survey respondents had a positive experience with telephone care following bariatric surgery and desired ongoing opportunities for virtual care. With its general acceptability by the public, telemedicine provides patients with convenient, time and cost-effective options for routine follow-up visits. The results generated from direct patient feedback support the need to integrate virtual care options into routine postoperative bariatric follow-up.

\section{Declarations}

Ethical Approval All procedures performed in studies involving human participants were in accordance with the ethical standards of the institutional and/or national research committee and with the 1964 Helsinki declaration and its later amendments or comparable ethical standards.

Conflict of Interest The authors declare no competing interests.

Informed Consent Informed consent was obtained from all individual participants included in the study.

\section{References}

1. Hintze JM, O'Riordan I, Hussain T, Keane E, Casserly P. Differences in patient satisfaction with virtual telephone clinics in a tertiary referral centre for otolaryngology during and after lockdown measures during the SARS-Cov2 pandemic. Ir J Med Sci. 2021;13:1-5.

2. Our World Data [Internet]. Policy responses to the coronavirus pandemic [cited $2021 \mathrm{Feb}$ 14]. Available from: https://ourworldin data.org/policy-responses-covid. Accessed 2 march 2021.

3. Canadian Institute for Health Information [Internet]. COVID-19's impact on health care systems; c2021 [cited 2021 Feb 14]. Available from: https://www.cihi.ca/en/covid-19-resources/impact-ofcovid-19-on-canadas-health-care-systems/overview-covid-19simpact-on. Accessed 2 march 2021.

4. Coldebella B, Armfield NR, Bambling M, Hansen J, Edirippulige S. The use of telemedicine for delivering healthcare to bariatric surgery patients: a literature review. J Telemed Telecare. 2018;24(10):651-60.

5. Nguyen M, Waller M, Pandya A, Portnoy J. A review of patient and provider satisfaction with telemedicine. Curr Allergy Asthma Rep. 2020;20(11):72.

6. Waller M, Stotler C. Telemedicine: a primer. Curr Allergy Asthma Rep. 2018;18(10):54.

7. Mayo Clinic [Internet]. Telehealth: technology meets healthcare; c2021 [cited 2021 Feb 15]. Available from: https://www.mayoc linic.org/healthy-lifestyle/consumer-health/in-depth/telehealth/ art-20044878. Accessed 2 march 2021.

8. Hincapié MA, Gallego JC, Gempeler A, Piñeros JA, Nasner D, Escobar MF. Implementation and usefulness of telemedicine 
during the COVID-19 pandemic: a scoping review. J Prim Care Community Health. 2020;11:1-7.

9. Kruse CS, Karem P, Shifflett K, Vegi L, Ravi K, Brooks M. Evaluating barriers to adopting telemedicine worldwide: a systematic review. J Telemed Telecare. 2018;24(1):4-12.

10. Jacklin KM, Henderson RI, Green ME, Walker LM, Calam B, Crowshoe LJ. Health care experiences of Indigenous people living with type 2 diabetes in Canada. CMAJ. 2017;189(3):E106-12.
11. Camp P, Girt M, Wells A, Malas A, Peter M, Crosbie S, et al. Virtual care for Indigenous populations in Canada, the United States, Australia, and New Zealand: protocol for a scoping review. JMIR Res Protoc. 2020;9(12):e21860.

Publisher's Note Springer Nature remains neutral with regard to jurisdictional claims in published maps and institutional affiliations. 